EPJ manuscript No.

(will be inserted by the editor)

\title{
Theoretical overview of atomic parity violation
}

\section{Recent developments and challenges}

\author{
Andrei Derevianko ${ }^{1}$ and Sergey G. Porsev ${ }^{2}$ \\ 1 Physics Department, University of Nevada, Reno, Nevada 89557, USA \\ 2 Petersburg Nuclear Physics Institute, Leningrad district, Gatchina, 188300, Russia \\ Received: date / Revised version: date
}

\begin{abstract}
Recent advances in interpreting the most accurate to-date measurement of atomic parity violation in Cs are reviewed. The inferred nuclear weak charge, $Q_{W}\left({ }^{133} \mathrm{Cs}\right)=-72.65(28)_{\text {expt }}(36)_{\text {theor }}$, agrees with the prediction of the standard model at $1 \sigma$ level. Further improved interpretation is limited by an accuracy of solving basic correlation problem of atomic structure. We report on our progress in solving this problem within the relativistic coupled-cluster formalism. We include single-, double- and triple- electronic excitations in the coupled-cluster expansion. Numerical results for energies, electric-dipole matrix elements, and hyperfine-structure constants of Cs are presented.
\end{abstract}

PACS. 32.80.Ys Weak-interaction effects in atoms - 31.15.Dv Coupled-cluster theory - 32.10.Fn Fine and hyperfine structure - 32.70.Cs Oscillator strengths, lifetimes, transition moments

\section{Introduction}

This year, year of 2006, marks 50 years of the fall of parity conservation, one of the hallmark discoveries of the 20th century physics. The field has started with the seminal Lee and Yang paper [1] and discovery of parity violation in the nuclear $\beta$-decay [2]. Soon after this discovery, Zel'dovich [3] contemplated possibility of observing paritynonconserving (PNC) signal in atoms. He concluded that the effect was too small to be of experimental significance. In 1974, however, M.-A. Bouchiat and C. Bouchiat [4] realized that the PNC is amplified in heavy atoms. They showed that the relevant PNC amplitude scales steeply with the nuclear charge $Z$. In atomic physics, the first P-violating signal has been observed in 1978 by Barkov and Zolotorev [5] in $\mathrm{Bi}$ atom. Over the following decades the experiments were refined, with PNC signal observed in several atoms. So far the most accurate measurement has been carried out in ${ }^{133} \mathrm{Cs}$ by Boulder group [6].

Rich history of atomic parity violation is examined in a number of review articles, e.g., Refs. [7, 8] and a book [9]. Here, due to the space limitation, we restrict our attention to recent developments in interpreting $\mathrm{P}$-violation in $\mathrm{Cs}$ atom and report on the progress towards more accurate interpretation of atomic PNC in this atom.

The PNC measurements are interpreted in terms of the weak nuclear charge $Q_{W}$, which quantifies the strength of the electroweak coupling between atomic electrons and quarks in the nucleus. The relation between $Q_{W}$ and the

Send offprint requests to: andrei@unr.edu
PNC amplitude, $E_{\mathrm{PNC}}$, can be represented as

$$
E_{\mathrm{PNC}}=k Q_{W},
$$

where $k$ is an atomic-structure factor. Apparently, the interpretation requires atomic-structure calculations of $k$ with an accuracy that matches the experimental uncertainty in $E_{\mathrm{PNC}}$. In particular, in Cs, the present theoretical uncertainty dominates over experimental error bar resulting in an overall $0.6 \%$ uncertainty in the inferred value of $Q_{W}\left({ }^{133} \mathrm{Cs}\right)$.

The underlying theory of the electroweak interactions is well established and its predictions have been verified in a variety of experiments. Much of the present efforts are driven by searches for "new physics" beyond the standard model (SM). We may distinguish between two approaches to such searches: low- and high-energy experiments. Atomic parity violation probes the low-energy electroweak sector of the SM. While the relevant momentum transfer is just $\sim 30 \mathrm{MeV}$, the exquisite accuracy of the interpretation constrains new physics at much higher energies. Indeed, following arguments of Ref. [10], we find that the present $0.6 \%$ determination of the $Q_{W}\left({ }^{133} \mathrm{Cs}\right)$ probes the new physics at a mass scale of

$$
\left\{8 \sqrt{2} \pi \frac{1}{G_{F}}\left|\frac{Q_{W}}{\delta Q_{W}}\right|\right\}^{1 / 2} \approx 20 \mathrm{TeV},
$$

where $G_{F}$ is the Fermi constant. Apparently by reducing the uncertainty in the determination of the weak charge, $\left|\delta Q_{W}\right|$, we may access even higher mass scales. Such a reduction in the uncertainty of $Q_{W}$ is the goal of our present efforts outlined in Sec. 3. 
Andrei Derevianko, Sergey G. Porsev: Theoretical overview of atomic parity violation

While discussing the impact of atomic PNC on particle physics, it is worth noting that colliders are blind to certain "new physics" scenarios [10]. For example, atomic $\mathrm{PNC}$ is uniquely sensitive to extra $\mathrm{Z}$ bosons predicted in grand unified theories, technicolor models, SUSY, and string theories. Generally, atomic PNC is one of a few probes of electroweak coupling below the $Z$-pole. Competing and at the same time complementary experiments include determination of the weak charge of electron at SLAC [11] and $\nu$-nucleus deep inelastic scattering by the $\mathrm{NuTeV}$ collaboration [12]. With an upgrade at Jefferson Lab, an even more accurate constraint on electroweak coupling is expected to come from a $\mathrm{P}$-violating $e-p$ scattering experiment (Qweak collaboration) [13]. Still, the atomic PNC will continue to play an important complementary role, as the atomic PNC is sensitive to electronneutron couplings, while the Qweak experiment will probe electron-proton coupling, i.e., a different linear combination in terms of the up and down quark-electron couplings.

\section{Deviation from the Standard Model and recent theoretical progress}

Parity violation in Cs has been a subject of a recent controversy. To infer the value of the weak charge, one needs to combine measurements with atomic-structure calculations. The uncertainty in the value of the inferred $Q_{W}$ is usually determined by summing experimental and theoretical uncertainties in quadrature,

$$
\sigma=\left\{\sigma_{\text {expt }}^{2}+\sigma_{\text {theor }}^{2}\right\}^{1 / 2} \text {. }
$$

In 1997 , the most accurate to date $(0.35 \%)$ measurement of PNC has been carried out by Boulder group [6]. At that time, the accuracy of calculations $[14,15]$ has been estimated to be $1 \%$. In 1999, Bennet and Wieman [16], in light of new lifetime data which improved theory-experiment agreement, have reduced the theoretical error bar to $0.4 \%$. While compared with the prediction of the SM, the resulting value of $Q_{W}$ differed by $2.5 \sigma$, one of the largest deviations in the recent history. The deviation has prompted a substantial interest from particle physics community (see, e.g., $[10,17,18,19])$. At the same time the reduced theoretical uncertainty raised the questions whether some "small" sub- $1 \%$ atomic-structure effects could be the reason for the deviation.

Over the last few years, there has been an important progress in understanding "small" corrections, such as Breit, radiative, and neutron-skin corrections. These advances are summarized in Table 1.

Taking into account the tabulated "small" corrections, revised value of vector transition polarizability and somewhat improved value of $E_{\mathrm{PNC}}$ [31], we arrive at the following revised value of the weak charge (the assigned theoretical uncertainty of $0.5 \%$ is consistent with an estimate of Ref. [31] and the central value with that of Ref. [29])

$$
Q_{W}\left({ }^{133} \mathrm{Cs}\right)=-72.65(28)_{\operatorname{expt}}(36)_{\text {theor }}
$$

This value agrees with the SM value [19] of $-73.19(13)$. at $1 \sigma$ level.

The present theoretical uncertainty in PNC calculations is estimated to be $0.5 \%$. Since the overall error is obtained with quadrature $(2)$, reducing $\sigma_{\text {theor }}$ below the experimental uncertainty of $0.35 \%$ will either reveal new physics or set important constraints on competing extensions to the SM. In addition, it will set a stage for further experimental efforts, such as those by Paris group $[32,33]$. Other experimental efforts which will benefit from the improved calculations are PNC measurements with $\mathrm{Ba}^{+}[34$, 35], Tl [36], and Fr [37].

\section{High-accuracy atomic-structure calculations}

The present accuracy in the determination of the weak charge of Cs is limited by the accuracy of solving the basic correlation problem. The many-body problem is hard. Even classically, the three-body problem can not be solved in closed form. While adequate numerical approaches (e.g., configuration interaction and variational methods) were developed for few-electron atoms, accurate solution of the many-body problem for atoms with many electrons still remains a challenge.

In a particular case of the PNC problem in Cs, we need to evaluate the PNC amplitude for the $6 S_{1 / 2} \rightarrow 7 S_{1 / 2}$ transition

$$
\begin{aligned}
& E_{\mathrm{PNC}}=\sum_{n} \frac{\left\langle 7 S|D| n P_{1 / 2}\right\rangle\left\langle n P_{1 / 2}\left|H_{W}\right| 6 S\right\rangle}{E_{6 S}-E_{n P_{1 / 2}}} \\
& +\sum_{n} \frac{\left\langle 7 S\left|H_{W}\right| n P_{1 / 2}\right\rangle\left\langle n P_{1 / 2}|D| 6 S\right\rangle}{E_{7 S}-E_{n P_{1 / 2}}}
\end{aligned}
$$

Here $D$ and $H_{\mathrm{W}}$ are electric-dipole and weak interaction matrix elements, and $E_{i}$ are atomic energy levels. The effective weak interaction, averaged over quarks is accumulated inside the nucleus

$$
H_{\mathrm{W}}=-\frac{G_{F}}{\sqrt{8}} Q_{W} \gamma_{5} \rho(\mathbf{r})
$$

where $\rho(\mathbf{r})$ is the neutron-density distribution. One could easily recast Eqs. (4) and (5) in terms of the structure factor $k$ of Eq. (1).

We wish to evaluate accurately the sum (4). Let us comment on pre-requisites for such calculations. (i) Due to the particular characters of the involved operators in summation (4), the approximate wave-functions have to reproduce the short-range (close to the nucleus) and long-range atomic properties simultaneously. The accurate short-range description requires ab initio relativistic approach, as the ratio of velocity of electron to the speed of light near the nucleus is $\sim \alpha Z \sim 0.4$ for Cs. (ii) With respect to the accuracy, we notice that simple Dirac-Hartree-Fock (DHF) calculations for the hyperfine-structure (HFS) constant of the ground state are about $50 \%$ off from the experimental value. This constant describes strength of coupling of the 
electron to the nuclear magnetic moment and its shortdistance nature mimics behavior of the weak matrix elements. Therefore we arrive at a conclusion that so-called correlation corrections (those beyond the DHF approximation) have to be addressed. We treat the correlations within the systematic and successful methods of manybody perturbation theory.

How do we estimate the accuracy of calculating $E_{\mathrm{PNC}}$ ? Ultra-precise experimental data are available for Cs. These data have accuracy better than our anticipated theoretical uncertainty thus allowing us to calibrate our calculations. Atomic energies are known to many significant figures. Ultra-precise ( $0.01 \%$ accurate) value of lifetime of the $6 P_{3 / 2}$ state of $\mathrm{Cs}$ has been recently inferred from photoassociation spectroscopy $[38,39]$ with ultracold atoms. The HFS constants for Cs are also measured to a good accuracy (the HFS constant $A_{6 S}$ of ${ }^{133} \mathrm{Cs}$ is known exactly by the definition of the second).

To reiterate, to further improve interpretation of atomic parity violating signals, we need a high-accuracy ab initio relativistic many-body method capable of reaching the accuracy level of $0.1 \%$ for Cs.

\section{Relativistic coupled-cluster method}

Many-body perturbation theory (MBPT) provides a systematic way of treating correlation corrections, the critical issue for an improved interpretation of atomic PNC. First we review main ideas of MBPT and the all-order coupledcluster (CC) method. Then we describe our present CCinspired computational scheme and illustrate it with numerical results for basic atomic properties of Cs atom.

\subsection{Generalities}

In MBPT the atomic Hamiltonian is partitioned as

$$
\begin{aligned}
H= & \left(\sum_{i} h_{\mathrm{nuc}}\left(\mathbf{r}_{i}\right)+\sum_{i} U_{\mathrm{DHF}}\left(\mathbf{r}_{i}\right)\right)+ \\
& \left(\frac{1}{2} \sum_{i \neq j} \frac{1}{r_{i j}}-\sum_{i} U_{\mathrm{DHF}}\left(\mathbf{r}_{i}\right)\right),
\end{aligned}
$$

where $h_{\text {nuc }}$ includes the kinetic energy of an electron and its interaction with the nucleus, $U_{\mathrm{DHF}}$ is the DHF potential, and the last term represents the residual Coulomb interaction between electrons. In MBPT the first part of the Hamiltonian is treated as the lowest-order Hamiltonian $H_{0}$ and the residual Coulomb interaction as a perturbation. The perturbative expansion is built in powers of residual interaction and the derivations typically involve methods of second quantization and diagrammatic techniques.

One of the mainstays of practical applications of MBPT is an assumption of convergence of series in powers of the residual interaction. Sometimes the convergence is poor and then one sums certain classes of diagrams to "all orders" using iterative techniques. One of the most popular all-order methods is the coupled-cluster (CC) formalism [40,41]. It is widely employed in atomic and nuclear physics, and quantum chemistry [42]. The relativistic atomic-structure CC-type calculations were carried out, for example, in Refs. [43,44,45,46,47,48].

The Hamiltonian in the second quantization (based on the DHF basis) reads

$$
\begin{aligned}
H & =H_{0}+G \\
& =\sum_{i} \varepsilon_{i}\left\{a_{i}^{\dagger} a_{i}\right\}+\frac{1}{2} \sum_{i j k l} g_{i j k l}\left\{a_{i}^{\dagger} a_{j}^{\dagger} a_{l} a_{k}\right\},
\end{aligned}
$$

where $H_{0}$ is the lowest-order Hamiltonian and the residual Coulomb interaction $G$ is treated as a perturbation. The operators $a_{i}$ and $a_{i}^{\dagger}$ are annihilation and creation operators, and $\{\ldots\}$ stands for a normal product of operators with respect to the core quasivacuum state $\left|0_{c}\right\rangle$. In the lowest order the atomic wave function with the valence electron in an orbital $v$ reads $\left|\Psi_{v}^{(0)}\right\rangle=a_{v}^{\dagger}\left|0_{c}\right\rangle$. For example, $v$ can represent the ground state $6 s$ orbital of Cs atom. Formally we can introduce a wave operator $\Omega$ that produces the exact many-body wave function, $\left|\Psi_{v}\right\rangle=\Omega\left|\Psi_{v}^{(0)}\right\rangle$.

Central to the $\mathrm{CC}$ method is realization that the wave operator can be represented via exponential ansatz [49]

$$
\Omega=\{\exp (K)\}=1+K+\frac{1}{2 !}\left\{K^{2}\right\}+\ldots .
$$

The operator $K$ can be compellingly separated into cluster operators combining simultaneous excitations of core and valence electrons from the reference state $\left|\Psi_{v}^{(0)}\right\rangle$ to all orders of MBPT,

$$
K=S+D+T+\cdots
$$

i.e., $K$ is separated into single $(S)$, double $(D)$, triple $(T)$, and higher-rank excitations. For the univalent systems it is convenient to subdivide cluster operators into core and valence classes

$$
K=K_{c}+K_{v}
$$

Clusters $K_{c}$ involve excitations from the core orbitals only, while $K_{v}$ describe simultaneous excitations of the core and valence electrons. Then $S=S_{c}+S_{v}, D=D_{c}+D_{v}$, etc. The cluster operators satisfy so-called Bloch equations [49].

While the CC formulation is exact, in practice the full cluster operator $K$ is truncated at a certain level of excitations. If we restrict it to single and double excitations

$$
K \equiv K^{(\mathrm{SD})} \approx S_{c}+D_{c}+S_{v}+D_{v}
$$

we arrive at the widely employed coupled-cluster single double (CCSD) method.

Linearized version of the CCSD method was employed by the Notre Dame group for high-accuracy calculations of various atomic properties $[43,44,47,48]$. In this approximation, one discards nonlinear terms in the expansion of exponent in Eq. $(7), \Omega \approx 1+K^{(\mathrm{SD})}$. We will refer to 
this approximation as the singles-doubles (SD) method. The resulting SD equations are written out in Ref. [43]. A typical $a b$ initio accuracy attained for properties of heavy alkali-metal atoms is at the level of $1 \%$.

Since the present goal is to reduce theoretical uncertainties to the level of $0.1-0.2 \%$ we have to go beyond the SD approach. A systematic step in improving the SD method would be an additional inclusion of triple and nonlinear double excitations. However, considering the present state of available computational power, the full incorporation of triples (specifically, core triples) seems to be unmanagable for heavy atoms. For instance, for Cs storing and manipulating core triple amplitudes would require $100 \mathrm{~Gb}$ of memory.

To motivate next-generation formalism, we have explicitly computed 1648 fourth-order diagrams for matrix elements that appear due to triple excitations and nonlinear terms (i.e., those omitted in the SD method) [50, 51]. We observe from numerical results for electric-dipole matrix elements in $\mathrm{Na}[51]$ and $\mathrm{Cs}$ [52] that the contributions from valence triples $T_{v}$ and nonlinear doubles $D_{n l}$ are much larger than those from core triples $T_{c}$. This leads to our present level of approximation: we discard core triples and core nonlinear terms and incorporate the valence triples and valence nonlinear terms into the SD formalism. The resulting approximation will be referred to as CCSDvT method.

\subsection{Driving equations in the CCSDvT approximation}

Below we write down the $\mathrm{CC}$ equations for cluster amplitudes in the CCSDvT approximation. Here we present topological structure of the equations only. A detailed tabulation of the formulas can be found in our paper [53]. The equations in the SD approximation are presented in explicit form in Ref. [43]. The CCSDvT equations for the core cluster amplitudes $S_{c}$ and $D_{c}$ are the same as in the SD approximation.

For valence triple amplitudes we obtain symbolically

$$
-\left[H_{0}, T_{v}\right]+\delta E_{v} T_{v} \approx T_{v}\left[D_{c}\right]+T_{v}\left[D_{v}\right] .
$$

Here $\left[H_{0}, T_{v}\right]$ is a commutator, and $\delta E_{v}$ is the correlation valence energy defined as

$$
\delta E_{v}=\delta E_{\mathrm{SD}}+\delta E_{\mathrm{CC}}+\delta E_{\mathrm{vT}}
$$

where correction $\delta E_{\mathrm{SD}}$ is obtained within the SD approach, correction $\delta E_{\mathrm{CC}}$ comes from nonlinear CC contributions and $\delta E_{\mathrm{vT}}$ is due to valence triples. Contributions $T_{v}\left[D_{c}\right]$ and $T_{v}\left[D_{v}\right]$ denote the effect of core and valence doubles on valence triples, respectively. At present we include only these effects omitting the effect of valence and core triples on valence triples $\left(T_{v}\left[T_{v}\right]\right.$ and $\left.T_{v}\left[T_{c}\right]\right)$ and nonlinear CC contributions. These are higher-order effects which computationally are much more demanding.
The topological structure of the valence singles equation is

$$
\begin{aligned}
& -\left[H_{0}, S_{v}\right]+\delta E_{v} S_{v} \approx \mathrm{SD}+ \\
& \quad S_{v}\left[S_{c} \otimes S_{v}\right]+S_{v}\left[S_{c} \otimes S_{c}\right]+ \\
& \quad S_{v}\left[S_{c} \otimes D_{v}\right]+S_{v}\left[S_{v} \otimes D_{c}\right]+S_{v}\left[T_{v}\right] .
\end{aligned}
$$

Here $S_{v}\left[S_{c} \otimes S_{v}\right]$ stands for a contribution from the excitations of core and valence electrons resulting from a product of clusters $S_{c}$ and $S_{v}$. All other terms are defined in a similar fashion.

Finally, equation for valence doubles can be symbolically represented as

$$
\begin{aligned}
& -\left[H_{0}, D_{v}\right]+\delta E_{v} D_{v} \approx \mathrm{SD}+ \\
& D_{v}\left[S_{c} \otimes S_{v}\right]+D_{v}\left[S_{c} \otimes S_{c}\right]+ \\
& D_{v}\left[S_{c} \otimes D_{v}\right]+D_{v}\left[S_{v} \otimes D_{c}\right]+D_{v}\left[S_{c} \otimes D_{c}\right]+ \\
& D_{v}\left[D_{c} \otimes D_{v}\right]+D_{v}\left[S_{c} \otimes T_{v}\right]+D_{v}\left[S_{v} \otimes T_{c}\right]+D_{v}\left[T_{v}\right] .
\end{aligned}
$$

Solution of the above equations provides us with the cluster amplitudes and correlation energies. Numerical results for the energies will be presented in Section 4.4. At this point, with the obtained wave functions we proceed to evaluating matrix elements.

\subsection{Matrix elements}

The SD method has already proven to be successful in calculations of various atomic properties. For heavy alkalimetal atoms the attained level of agreement with experimental data for the hyperfine constants is at $5 \%$ and the accuracy of a similar calculation for the electric-dipole amplitudes is $0.5 \%$ (see, e.g., [48]). At the same time the accuracy required for our goals should be at the level of $0.1-0.2 \%$. In order to improve the overall accuracy we develop the technique of relativistic calculations of matrix elements beyond the SD approach.

Given two computed CCSDvT wave functions, we may evaluate matrix elements of one-electron operator $Z$ as

$$
Z_{w v}=\frac{\left\langle\Psi_{w}\left|\sum_{i j} z_{i j} a_{i}^{\dagger} a_{j}\right| \Psi_{v}\right\rangle}{\sqrt{\left\langle\Psi_{w} \mid \Psi_{w}\right\rangle\left\langle\Psi_{v} \mid \Psi_{v}\right\rangle}} .
$$

The explicit expressions are given in Ref. [53]. Compared to the SD approximation, we include contribution of valence triples $T_{v}$. They contribute both directly via explicit contributions to matrix element formula and indirectly through modification of the SD amplitudes.

It is worth pointing out, that the importance of the valence triples has been realized earlier by the Notre Dame group [44]. They have shown that at the SD level, the error for the HFS constants is as large as $5 \%$ for Cs. To rectify this problem, they proposed and implemented a scheme that approximates the effect $S_{v}\left[T_{v}\right]$, i.e., effect of valence triples on valence singles. While improving agreement for the HFS constants, their approximation leads to poorer agreement (compared to SD method) for the dipole matrix elements. The advantage of the Notre Dame scheme is 
that it avoided expensive storing of triple excitations. Due to improved computational resources, we are able to store triples. Accounting for the triples in a rigorous fashion leads to a better agreement between theory and experiment.

Compared to the Notre Dame approximation we also include dressing of matrix elements based on the $\mathrm{CC}$ ansatz. The idea of our method[52] is as follows. When the CC exponent is expanded in Eq. (15), we encounter infinite number of terms. We devised a method of partial summation (dressing) of the resulting series. Our formalism is built upon an expansion of the product of cluster amplitudes into a sum of $n$-body insertions. We considered two types of insertions: particle (hole) line insertion (line "dressing") and two-particle (two-hole) random-phase-approximationlike insertion. We demonstrated how to "dress" these insertions and formulated iterative equations.

Another formal improvement over Notre Dame calculations comes from including the $\mathrm{CC}$ nonlinear terms in the equations for valence singles (13) and doubles (14). We also include contribution of the core triples to matrix elements from direct fourth-order calculation.

\subsection{Numerical results}

Our developed numerical CCSDvT code is an extension of the relativistic SD code [47] which employs B-spline basis set. This basis numerically approximates complete set of single-particle atomic states. Here we use 35 out of 40 positive-energy basis functions. Basis functions with $l_{\max } \leq 5$ are used for singles and doubles. For triples we employ a more limited set of basis functions with $l_{\max }\left(T_{v}\right) \leq$ 4. Excitations from core sub-shells $[4 s, \ldots, 5 p]$ are included in the calculations of triples while excitations from subshells $[1 s, \ldots, 3 d]$ are discarded.

Table 2. Contributions to removal energies of $6 s, 6 p_{1 / 2}$, and $6 p_{3 / 2}$ states for $\mathrm{Cs}$ in $\mathrm{cm}^{-1}$ in various approximations. $\delta E_{\text {extrapolated }}^{\text {tot }}$ correction is obtained by computing SD properties with increasingly larger basis sets and interpolating them to $l=\infty$ [54]. A comparison with experimental values is presented in the lower panel.

\begin{tabular}{lrrr}
\hline \hline & \multicolumn{1}{c}{$6 s$} & $6 p_{1 / 2}$ & $6 p_{3 / 2}$ \\
\hline$E_{\mathrm{DHF}}$ & 27954 & 18790 & 18389 \\
$\delta E_{\mathrm{SD}}$ & 3869 & 1611 & 1623 \\
$\delta E_{\mathrm{CCSDvT}}$ & 3350 & 1387 & 1220 \\
$E_{\mathrm{CCSDvT}}^{\text {tot }}$ & 31304 & 20178 & 19608 \\
$\mathrm{QED}^{1}$ & 18 & -0.4 & 0 \\
$\delta E_{\text {extrapolated }}^{\text {tot }}$ & 30 & 20 & 20 \\
\hline $\mathrm{E}_{\text {final }}^{\text {tot }}$ & 31352 & 20198 & 19628 \\
$E_{\text {experim }}{ }^{2}$ & 31407 & 20228 & 19675 \\
\hline \hline
\end{tabular}

${ }^{1}$ Reference [28]; ${ }^{2}$ Reference [55].

Computed removal energies of $6 s, 6 p_{1 / 2}$, and $6 p_{3 / 2}$ states of atomic cesium are presented in Table 2 . The dom- inant contribution to the energies comes from the DHF values. The remaining (correlation) contribution is given by Eq. (12). We computed this correlation correction in SD and CCSDvT approximations. As it follows from the table the agreement with experiment is at the level of 0.1 $0.2 \%$ for all considered states. We anticipate that including other corrections missed at this stage (e.g., nonlinear corrections to core amplitudes and core triples) can further improve the agreement with the experimental results.

With the computed wave functions of the $6 s, 6 p_{1 / 2}$ and $6 p_{3 / 2}$ states we determine matrix elements. Numerical results for magnetic-dipole hyperfine-structure constants $A$ and electric-dipole transition amplitudes are presented in Table 3. This Table is organized as follows. First we list the DHF and SD values, and the differences between CCSDvT and SD values, $\Delta($ CCSDvT $)=$ CCSDvT $-\mathrm{SD}$. We base our final $a b$ initio results on the most sophisticated CCSDvT values. These values also include all-order dressing, and corrections due to core triples, computed in the the fourth order of MBPT. QED corrections are included where available. The results for the HFS constants include finite-nuclear size (Born-Weisskopf) effect.

We find an excellent, 0.1\%-level, agreement for dipole matrix elements and the HFS constant of the ground state. The agreement for the the HFS constant of the $6 p_{1 / 2}$ is only at $1 \%$ level. We are presently working on testing sensitivity of this constant to higher-order effects.

We would like to emphasize that presently the correlation corrections at the level of a few $0.1 \%$ are comparable to radiative corrections. In this regard it would be useful to carefully compute them to unmask the remaining many-body effects.

\section{Summary and outlook}

Atomic parity violation plays an important role in testing low-energy electroweak sector of standard model. Interpretation of experiments in terms of nuclear weak charge requires calculations challenging the capabilities of modern atomic theory. Over the last few years, we have witnessed a substantial advance in evaluating corrections to parity violating amplitudes in heavy atoms. These small (sub$1 \%$ ), but important corrections include Breit, radiative (vacuum polarization, self-energy, and vertex) and neutron skin corrections. As a result of this progress, the most accurate to date measurement of atomic parity violation in Cs has been brought into substantial agreement with the prediction of the standard model.

Presently the theoretical interpretation is clouded by uncertainties in solving the basic correlation problem of atomic structure. In this paper we outlined our next-generation many-body formalism for solving this problem. We tested our coupled-cluster-inspired method by computing basic atomic properties of Cs atom. All the computed properties are important for quantifying accuracy of the calculations of parity-violating amplitudes. We find an agreement at $0.1 \%$ for the ground state hyperfine structure constant, E1 transition amplitudes, and energies. 
However, a relatively poor $1 \%$ agreement of the HFS constant $A$ for the $6 P_{1 / 2}$ state with experiment requires further improvements of the method. The advantage of the employed coupled-cluster method is that it allows for such systematic improvements. It is anticipated that the further theoretical progress will refine constraints on new physics beyond the standard model and enable next round of experimental studies.

This work was supported in part by the US National Science Foundation, by the US NIST precision measurement grant, and by the Russian Foundation for Basic Research under Grant Nos. 04-02-16345-a and 05-02-16914-a. This manuscript was completed with a partial support by NSF through a grant for the Institute for Theoretical Atomic, Molecular, and Optical Physics at Harvard University and Smithsonian Astrophysical Observatory.

\section{References}

1. T.D. Lee, C.N. Yang, Phys. Rev. 104, 254 (1956)

2. C.S. Wu, E. Ambler, R.W. Hayward, D.D. Hoppes, R.P. Hudson, Phys. Rev. 105, 1413 (1957)

3. Ya.B. Zel'dovich, Zh. Eksp. Teor. Fiz. 36, 964 (1959), [Sov. Phys.-JETP 9 681, (1959)]

4. M.A. Bouchiat, C. Bouchiat, J. Phys. 35, 899 (1974)

5. L.M. Barkov, M.S. Zolotorev, Pis'ma Zh. Eksp. Teor. Fiz. 27, 379 (1978), [JETP Lett. 27, 357 (1978)]

6. C.S. Wood, S.C. Bennett, D. Cho, B.P. Masterson, J.L. Roberts, C.E. Tanner, C.E. Wieman, Science 275, 1759 (1997)

7. M.A. Bouchiat, C. Bouchiat, Rep. Prog. Phys. 60, 1351 (1997)

8. J.S.M. Ginges, V.V. Flambaum, Phys. Rep. 397, 63 (2004)

9. I.B. Khriplovich, Parity non-conservation in atomic phenomena (Gordon and Breach, New York, 1991)

10. M.J. Ramsey-Musolf, Phys. Rev. C 60, 015501 (1999)

11. P.L. Anthony, R.G. Arnold, C. Arroyo, K. Bega, J. Biesiada, P.E. Bosted, G. Bower, J. Cahoon, R. Carr, G.D. Cates et al., Phys. Rev. Lett. 95, 081601 (2005)

12. G.P. Zeller, K.S. McFarland, T. Adams, A. Alton, S. Avvakumov, L. de Barbaro, P. de Barbaro, R.H. Bernstein, A. Bodek, T. Bolton et al., Phys. Rev. Lett. 88, 091802 (2002)

13. Homepage of QWeak collaboration at Jefferson Lab, www.jlab.org/qweak/

14. V.A. Dzuba, V.V. Flambaum, O.P. Sushkov, Phys. Lett. A 141, 147 (1989)

15. S.A. Blundell, W.R. Johnson, J. Sapirstein, Phys. Rev. Lett. 65, 1411 (1990), Phys. Rev. D 45, 1602 (1992)

16. S.C. Bennett, C.E. Wieman, Phys. Rev. Lett. 82, 2484 (1999)

17. R. Casalbuoni, S. de Curtis, D. Dominici, R. Gatto, Phys. Lett. B 460, 135 (1999)

18. J.L. Rosner, Phys. Rev. D 61, 016006 (2000)

19. J.L. Rosner, Phys. Rev. D 65, 073026 (2002)

20. A. Derevianko, Phys. Rev. Lett. 85, 1618 (2000)

21. V.A. Dzuba, C. Harabati, W.R. Johnson, M.S. Safronova, Phys. Rev. A 63, 044103 (2001)

22. M.G. Kozlov, S.G. Porsev, I.I. Tupitsyn, Phys. Rev. Lett. 86, 3260 (2001)
23. W.R. Johnson, I. Bednyakov, G. Soff, Phys. Rev. Lett. 87, 233001 (2001)

24. M. Kuchiev, V. Flambaum, Phys. Rev. Lett. 89, 283002 (2002)

25. A.I. Milstein, O.P. Sushkov, I.S. Terekhov, Phys. Rev. Lett. 89, 283003 (2002)

26. A. Milstein, O. Sushkov, I. Terekhov, Phys. Rev. A 67, $62103(2003)$

27. J. Sapirstein, K. Pachucki, A. Veitia, K.T. Cheng, Phys. Rev. A 67, 052110 (2003)

28. V.V. Flambaum, J.S.M. Ginges, Phys. Rev. A 72, 052115 (2005)

29. V.M. Shabaev, K. Pachucki, I.I. Tupitsyn, V.A. Yerokhin, Phys. Rev. Lett. 94, 213002 (2005)

30. A. Derevianko, Phys. Rev. A 65, 012106 (2002)

31. V.A. Dzuba, V.V. Flambaum, J.S.M. Ginges, Phys. Rev. D 66, 076013 (2002)

32. J. Guena, D. Chauvat, P. Jacquier, E. Jahier, M. Lintz, S. Sanguinetti, A. Wasan, M.A. Bouchiat, A.V. Papoyan, D. Sarkisyan, Phys. Rev. Lett. 90, 143001 (2003)

33. J. Guena, M. Lintz, M.A. Bouchiat, Phys. Rev. A 71, $042108(2005)$

34. N. Fortson, Phys. Rev. Lett. 70, 2383 (1993)

35. T.W. Koerber, M.H. Schacht, W. Nagourney, E.N. Fortson, J. Phys. B 36, 637 (2003)

36. P.A. Vetter, D.M. Meekhof, P.K. Majumder, S.K. Lamoreaux, E.N. Fortson, Phys. Rev. Lett. 74, 2658 (1995)

37. E. Gomez, L.A. Orozco, G.D. Sprouse, Rep. Prog. Phys. 69, 79 (2006)

38. C. Amiot, O. Dulieu, R.F. Gutterres, F. Masnou-Seeuws, Phys. Rev. A 66, 052506 (2002)

39. N. Bouloufa, A. Crubellier, O. Dulieu, (private communications)

40. F. Coester, H.G. Kümmel, Nucl. Phys. 17, 477 (1960)

41. J. Čižek, J. Chem. Phys. 45, 4256 (1966)

42. R.F. Bishop, H.G. Kümmel, Physics Today 3, 52 (1987)

43. S.A. Blundell, W.R. Johnson, Z.W. Liu, J. Sapirstein, Phys. Rev. A 40, 2233 (1989)

44. S.A. Blundell, W.R. Johnson, J. Sapirstein, Phys. Rev. A 43, 3407 (1991)

45. E. Eliav, U. Kaldor, Y. Ishikawa, Phys. Rev. A 50, 1121 (1994)

46. E.N. Avgoustoglou, D.R. Beck, Phys. Rev. A 57, 4286 (1998)

47. M.S. Safronova, A. Derevianko, W.R. Johnson, Phys. Rev. A 58, 1016 (1998)

48. M.S. Safronova, W.R. Johnson, A. Derevianko, Phys. Rev. A 60, 4476 (1999)

49. I. Lindgren, J. Morrison, Atomic Many-Body Theory, 2nd edn. (Springer-Verlag, Berlin, 1986)

50. A. Derevianko, E.D. Emmons, Phys. Rev. A 66, 012503 (2002)

51. C.C. Cannon, A. Derevianko, Phys. Rev. A 69, 030502(R) (2004)

52. A. Derevianko, S.G. Porsev, Phys. Rev. A 71, 032509 (2005)

53. S.G. Porsev, A. Derevianko, Phys. Rev. A 73, 012501 (2006)

54. M.S. Safronova, Ph.D. thesis, University of Notre Dame (2000)

55. C.E. Moore, Atomic energy levels, Vol. III (National Bureau of Standards, Washington, D.C., 1958)

56. J. Sapirstein, K.T. Cheng, Phys. Rev. A 67, 022512 (2003)

57. R.J. Rafac, C.E. Tanner, Phys. Rev. A 56, 1027 (1997)

58. R.J. Rafac, C.E. Tanner, Phys. Rev. A 58, 1087 (1998) 


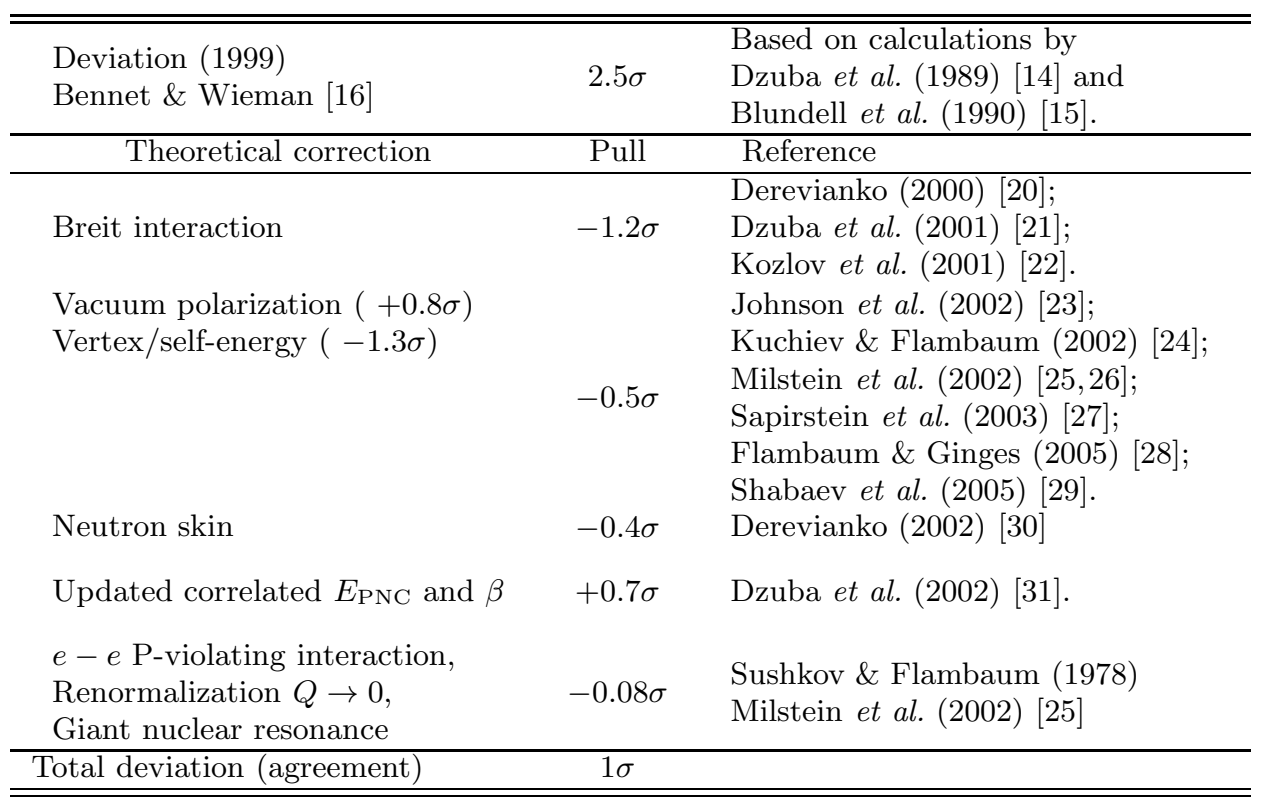

Table 1. Present status of the $2.5 \sigma$ deviation of inferred $Q_{W}\left({ }^{133} \mathrm{Cs}\right)$ from the prediction of the Standard Model. In the bulk of the Table, we summarize the recent theoretical progress on computing "small" sub-1\% corrections to the $6 s-7 s$ PNC amplitude. These corrections directly affect the inferred value of the weak charge of Cs. For illustrative purposes all the corrections (pulls) are tabulated in terms of the original Bennet and Wieman [16] uncertainty, $\sigma=\left\{\sigma_{\text {expt }}^{2}+\sigma_{\text {theor }}^{2}\right\}^{1 / 2} \approx 0.5 \%$.

Table 3. Magnetic-dipole hyperfine structure constants $A$ (in $\mathrm{MHz}$ ) and matrix elements of electric dipole moment (in a.u.) for ${ }^{133} \mathrm{Cs}$. Results of calculations and comparison with experimental values are presented. See text for the explanation of entries.

\begin{tabular}{lrccc}
\hline \hline & $A(6 s)$ & $A\left(6 p_{1 / 2}\right)$ & $\left\langle 6 p_{1 / 2}\|D\| 6 s\right\rangle$ & $\left\langle 6 p_{3 / 2}\|D\| 6 s\right\rangle$ \\
\hline DHF & 1425.4 & 160.94 & 5.2777 & 7.4264 \\
SD & 2438.0 & 310.71 & 4.4829 & 6.3075 \\
$\Delta$ (CCSDvT) & -136.9 & -20.92 & 0.0256 & 0.0363 \\
\multicolumn{1}{l}{ Complementary } & corrections & & \\
Line dressing & -12.5 & -2.16 & 0.0094 & 0.0107 \\
Vertex dressing & 4.3 & 0.29 & -0.0067 & -0.0088 \\
MBPT-IV (core triples,...) & 7.8 & 1.14 & 0.0001 & 0.0001 \\
Breit + QED & $-6.5^{1}$ & & $0.0024^{2}$ &. \\
Extrapol. for $l=\infty$ & 5.0 & 0.37 & -0.004 & -0.006 \\
Final CCSDvT + corrections & 2299.2 & 289.43 & 4.5097 & 6.3398 \\
Experiment & 2298.2 & $291.89(8)$ & $4.5049(17)^{5}$ & $6.3404(3)^{6}$ \\
\hline \hline
\end{tabular}

${ }^{1}$ Ref. [56]; ${ }^{2}$ Refs. [20,28]; ${ }^{3}$ Ref. [54]; ${ }^{4}$ Ref. [57]; ${ }^{5}$ Refs. [58,39]; ${ }^{6}$ Ref. [39]. 